\title{
Surgical management of adrenal cysts: a single-institution experience
}

\author{
Xiao Lyu, Liangren Liu, Lu Yang, Liang Gao, Qiang Wei \\ Department of Urology, West China Hospital, Sichuan University, Chengdu, Sichuan, P.R. China
}

\section{ABSTRACT}

Objective: To analyze surgical methods and evaluate treatment efficacy and safety for managing adrenal cystic lesions.

Materials and methods: All patients presenting with adrenal lesions of the West China Hospital were reviewed retrospectively from January 2003 to April 2013 and 47 were diagnosed as adrenal cysts. Basic information, clinical history, physical examination, laboratory investigations, abdominal ultrasound and enhanced computed tomography were detailed noted. Cysts with different surgical management were analyzed and surgery option operative time, postoperative complications and after-surgery hospital stay were all noted. The final diagnosis was judged by histopathology. Patients were followed from 3 month to 10 years.

Results: All the 47 patients with a mean age of 43.8 years were managed by surgical intervention. Compared laparoscopic technology with open technology, the laparoscopic has the advantage of a shorter operation time, shorter hospital stay after surgery and enhanced cosmesis. The histopathologic result was: 23 (50\%) were endothelial cysts and 16 (35\%) were pseudocysts. One patient had evidence to recurrence at the followed-up stage.

Conclusion: Adrenal cysts are rare and with the development of imaging techniques many of these are diagnosed incidentally. CT has advantages in detecting the cysts with haemorrhage, intracystic debris, calcification and mixed adrenal mass. Minimally invasive surgery offers equivalent efficacy to traditional open procedures, while providing a shorter operation time, shorter convalescence and improved cosmesis. Patients after surgical resection should be followed up closely especially if functional cysts and histopathology of cystic tumor are present.

\section{ARTICLE INFO}

\section{Key words:}

Laparoscopy; Cysts;

Endothelium; Adrenal Rest

Tumor

Int Braz J Urol. 2014; 40: 656-65

Submitted for publication:

December 19, 2013

Accepted after revision:

March 19, 2014

\section{INTRODUCTION}

Adrenal cystic lesions (ACL) are rare. Since the first report of Greiselius in 1670, over 600 adrenal cysts have been reported in literatures $(1,2)$. The occurrence of ACL by autopsy is about $0.064 \sim 0.18 \%$ of general people (3).The incidence rate increases with the development of diagnostic methods like ultrasound(US), computed tomography (CT) and magnetic resonance imaging (MRI) (3). Most ACL are unilateral, while 8 15\% are bilateral without obvious side predominance. ACL can be seen at any age especially between 30 s and 60 s (3). Most adrenal cysts are nonfunctioning and benign, while some literatures indicate that adrenal cysts may be malignant with an incidence of 
$7 \%(2,4-7)$. The pathogenesis is still unclear and several hypotheses have been proposed $(3,7)$. There is controversy concerning the recommendation of adrenal cyst management because of low incidence, difficult pre-surgery pathologic diagnosis and various surgical intervention ways (6-8). The previous questions made us to review patients with adrenal cysts with different clinical characteristics who were managed in different ways.

\section{MATERIALS AND METHODS}

We retrospectively reviewed the patients with adrenal lesions from the Urology Department, West China Hospital from January 2003 to April 2013. 47 patients were diagnosed with adrenal cysts whose basic information such as age, gender and location of cystic lesion were recorded. Each patient had a brief clinical history and physical examination. Abdominal US was used as first choice to screen the cysts. Then abdominal enhanced CT scans were provided for all patients. MRI was used for two patients only. The diameter of cysts was measured by CT scans.

Serum cortisol at $8 \mathrm{am}, 4 \mathrm{pm}$ and $24 \mathrm{pm}$ points, 24-hour urinary catecholamine levels and serum catecholamine, aldosterone in both lying position and standing position, serum metanephrine and potassium were analyzed to evaluate the hormonally active adrenal cysts. 46 patients chose surgical removal and one underwent percutaneous FNA (percutaneous fine-needle aspiration) mediated by CT. Surgical resection were taken in our series in patients with the following characteristics: hormonally active adrenal cysts, cystic lesion greater than $4 \mathrm{~cm}$, smaller cysts enlarged at least by $1 \mathrm{~cm}$ per year, cysts with image changing suggesting haemorrhage and calcification. Patients out of these standards were treated with watchful waiting with abdominal enhanced CT scans provided at the urological outpatient service every 6 months. Surgery option, operative time, postoperative complications and after-surgery hospital stay were all detailed noted. In all open cases the procedure was performed using waist incision methods and all laparoscopy cases were submitted to retroperitoneal laparoscopy. The final diagnosis was obtained according to the histopathologic result of specimen obtained from surgical resection of surgical patients.

All the patients after surgery were followed from 3 months to 10 years as urological outpatients every 3 months in the first year. If patients showed no evidence of recurrence, we changed it to every 6 months in the second year. After that, if there was no positive found, we changed it to every one year in the next years.

SPSS19.0 (IBM, Chicago, USA) was used for statistical analysis. Independent samples t-test was used to compare the laparoscopy surgery with open surgery.

\section{RESULTS}

Among 922 patients operated on for adrenal lesions in the ten years period in the Urology Department, West China Hospital, cystic lesions were found in 47 patients, accounting for 5.1\% of adrenal lesions. The age ranged from 18 to 77 with a mean age of 43.8. $29(61.7 \%)$ of them were female and the rest 18 (38.3\%) were male. All cysts were unilateral with 26 (55.3\%) at left side and $21(44.7 \%)$ at right side (Table-1). 12 of them presented with lower back pain and 2 complained of abdominal pain with abdominal symptoms. 3 masses could be obviously palpated. 6 cysts were discovered incidentally by non-specific symptoms like headache, palpitations, fever, hypertension and ureteral calculi. 27 of them were asymptomatic and detected by conventional medical examination. US detected 38 adrenal cysts, 3 adrenal masses, 2 kidney cysts and 1 hepatic cyst with 3 patients not reported. CT detected 42 adrenal cysts, 4 adrenal masses and 1 hepatic cyst. MRI detected 2 adrenal cysts. The mean diameter of the cysts measured by CT was $5.0 \mathrm{~cm}$, ranging from $1.4 \mathrm{~cm}$ to $20 \mathrm{~cm}$. Among 4 patients with functional tumors, 2 had hypertension with elevated level of 24-hour urinary catecholamine and 2 had hypertension and hyperaldosteronism.

All 47 patients were managed by surgical intervention, wherein 1 underwent FNA mediated by CT, 8 had cysts excisions with cysts excised and adrenal gland preserved, 5 had adrenalectomies, 8 had laparoscopic marsupialization 
Table 1 - Features of Patients with Adrenal cysts and pathological examination result.

\begin{tabular}{lc}
\hline Number & 47 \\
\hline Mean age(year) & $43.8(18-77)$ \\
Mean size(cm) & $5.0(1.4-12)$ \\
Male (n) & $18(38.3 \%)$ \\
Female(n) & $29(61.7 \%)$ \\
Right side(n) & $21(44.7 \%)$ \\
Left side(n) & $26 \%(55.3 \%)$ \\
Histologic type (n) & 46 \\
Endothelial cysts(n) & $23(50 \%)$ \\
Pseudocysts(n) & $16(36 \%)$ \\
Pheochromocytomas cyst(n) & $2(4 \%)$ \\
Cystic adenomas(n) & $2(4 \%)$ \\
Epithelial cyst(n) & $2(4 \%)$ \\
Bronchogenic cyst(n) & $1(2 \%)$ \\
Pulmonary sequestration(n) & $1(2 \%)$ \\
\hline
\end{tabular}

or decortication, 21 had laparoscopic cysts excisions and 4 had laparoscopic adrenalectomies. One patient proposed to receive laparoscopic cyst excision was immediately replaced by open cyst excision, as there were difficulties in building retroperitoneal laparoscopic passageway. Another patient who had a laparoscopic cyst excision was found the cyst reappeared (about $2 \mathrm{~cm}$ ) in the next 3 months. Thus an open adrenalectomy was chosen, and during the operation the inferior vena cava was inadvertently injured and was success- fully repaired. One patient who had adrenal cyst with ureteral calculi had laparoscopic adrenalectomy and holmium laser lithotripsy simultaneously. Therefore, the 3 patients were excluded in the comparison of the open and laparoscopic technique (Table-2). The mean cysts size, operative time and after-surgery hospital stay between the open and laparoscopic techniques were 6.55 and $4.66 \mathrm{~cm}(\mathrm{p}=0.081), 95$ and $62.44 \mathrm{~min}(\mathrm{p}<0.001)$, and 6.09 and 4.47 days $(p=0.006)$ respectively. There were no significant complications in the postoperative period in all 47 patients.

Histopathologic result of the 46 patients was as follow: 23 was endothelial cysts, 16 pseudocysts, 2 cystic pheochromocytomas, 2 cystic adenomas, 1 epithelial cyst, 1 bronchogenic cyst and 1 pulmonary sequestration cyst (Table-1). Calcifications within the cysts were found in 7 patients. Only one patient had evidence of recurrence at the followed-up stage. 10 patients presented with lower back pain and 2 complained of abdominal pain and both disappeared after surgery. However, 2 patients complained their lower back pain still existed, which we suspected was due to lumbar muscle strain.

\section{DISCUSSION}

Cystic lesions in the adrenal gland are uncommon (a reported incidence at autopsy of $0.064 \%$ to $0.18 \%$ ). Only few large series have been reported and our study is one of the largest studies with 47 cases $(3,6,9,10)$. Incidence of adrenal cysts in adrenal disease was 5.1\%, according to various clinical series of $5.4 \%(11,12)$.The average age of patients was 43.8 years, slightly

Table 2 - The demographic and clinical data of surgical intervention patients.

\begin{tabular}{lcccc}
\hline Variable & Open surgery & Laparoscopy & $p$ & $95 \% \mathrm{Cl}$ \\
\hline $\mathrm{n}$ & 11 & 32 & & \\
age & 44.64 & 44.84 & 0.967 & 0.081 \\
Cyst size,cm & 6.55 & 4.66 & $<0.001$ & {$[-9.72,10.14]$} \\
Operative time, min & 95.00 & 62.44 & 0.006 & {$[-49.28,-15.84]$} \\
after-surgery hospital stay & 6.09 & 4.47 & {$[-2.74,-0.50]$} \\
\hline
\end{tabular}

$\mathbf{C I}=$ confidence interval 
higher than previously reported $(6,12)$ because our center deals only with adult patients. Adrenal cysts have been reported to be more common in women. In our study, the male to female ratio was 1:1.6 $(1,13)$. All cysts were unilateral and appeared almost equally in both sides although epidemiology indicated $8 \sim 15 \%$ are bilateral. 27 of the 47 cases (57\%) were discovered asymptomatic by health examination. 6 cysts were discovered incidentally by non-specific symptoms like headache, palpitations, fever, hypertension and ureteral calculi. Abdominal or flank pain, gastrointestinal symptoms and a palpable mass were the main clinical manifestations. Large adrenal cysts adjacent to abdominal structures are typical examples. 14 patients (30\%) complained of abdominal pain or gastrointestinal symptoms, wherein 3 cases of adrenal cysts could be palpated obviously. The mean size of symptomatic cysts was larger than that of asymptomatic cysts (7.2 cm vs. $4.4 \mathrm{~cm})$. Large cysts have a tendency to develop such complications as intracystic hemorrhage and rupture that can manifest as a surgical emergency; however, no case was observed in our study (14-17). Functioning cysts of the adrenal cortex and medulla have been reported (9). Two cystic pheochromocytomas and two cystic adenomas were found. Cystic pheochromocytomas presented hypertension, headache and palpitations. Laboratory test showed high values of 24-hour urinary catecholamine. Cystic adenomas presented hypertension and hyperaldosteronism.

US, CT and MRI are major modalities used in the evaluation of adrenal cysts. In the present series, the sensitivity of US for adrenal cyst was $89 \%$ and of CT was 98\%. US can easily find the borders of the cysts and identify uncomplicated cysts, however, it had limitation in distinguishing the source of retroperitoneal cysts and adrenal mass accompanied with cysts (18). In our series the US mislead adrenal cysts as renal cysts or hepatic cysts. And it had low sensitivity for detection of small masses compared with CT and MRI. The advantage of CT was to detect the cysts with haemorrhage, intracystic debris and calcification (18). Meanwhile, CT was good for mixed adrenal mass (7). On CT, uncomplicated benign adrenal cysts are of water density (0-20 HU).
Higher attenuation values can be observed due to haemorrhage, intracystic debris and calcification (18). The CT misdiagnosed an adrenal cyst as a hepatic cyst due to its $20 \mathrm{~cm}$ diameter and unclear border with the liver. Some literatures found that CT may suggest malignancy through calcification, thickness and enhancement of the vesicle wall (Figure-1) $(18,19)$. It requires more samplings and thorough histopathologic examination. We only had two cases diagnosed by MRI so it proved with low reference value.

Cysts of the adrenal gland have been classified traditionally into four categories by origin: endothelial cyst, 45\%; pseudocyst, 39\%; epithelial cyst, 9\%; parasitic cyst, 7\% (Figure-2) $(6,17$, 20). However, Neri and Nance found pseudocyst represented the most common subtype (2). Among the 46 cases, 23 (50\%) were endothelial cysts, 16 (35\%) were pseudocysts and 1 (2\%) was an epithelial cyst. No parasitic cysts were identified. Endothelial cysts can also be subcategorized by histology into lymphangiomatous and angiomatous cysts. They are distinguished from tumors by the absence of proliferating endothelium. The pathogenesis of adrenal pseudocyst is unclear. Most scholars think pseudocysts result from the organization of a previous traumatic hematoma or infectious process $(7,9,21)$.The epithelial cyst is rare and only one example was found. Its pathogenesis is still unclear and can be subdivided into glandular or retention cysts, cystic adenomas and embryonal cysts $(7,22)$. In our series, a bronchogenic cyst and a pulmonary sequestration cyst were found in the adrenal region. Bronchogenic cyst is a rare developmental abnormality of the primitive foregut that arises during early embryogenesis from abnormal budding of the developing tracheobronchial tree (23). Pulmonary sequestration cyst is extremely rare and is characterized by a mass of nonfunctional lung tissue without communication with the bronchopulmonary tree and fed from an aberrant systemic artery (24). In both bronchogenic cyst and pulmonary sequestration cyst, histology still remains the routine method of diagnosis and surgery remains the predominant therapeutic option $(23,24)$.

Surgical intervention was the main management method for adrenal cyst with several 
Figure 1 - A) Adrenal endothelial cyst. Contrast-enhanced computed tomography scan of abdomen showing a cystic lesion measuring $7.9 \times 9.3 \mathrm{~cm}$ in size at the left adrenal area. B) Adrenal pseudocyst. Contrast-enhanced computed tomography scan of abdomen showing a cystic lesion surrounded by calcification measuring $3.3 \times 3.0 \mathrm{~cm}$ in size at the left adrenal area. C) Adrenal lymphangiomatous cyst. Contrast-enhanced computed tomography scan of abdomen showing a cystic lesion with several punctuate calcification measuring $1.8 \times 1.3 \mathrm{~cm}$ in size at the right adrenal area. D) Recurrence of adrenal cyst. Contrast-enhanced thinner computed tomography scan of abdomen showing a cystic lesion measuring $2 \times 1.5 \mathrm{~cm}$ in size at the right adrenal area.
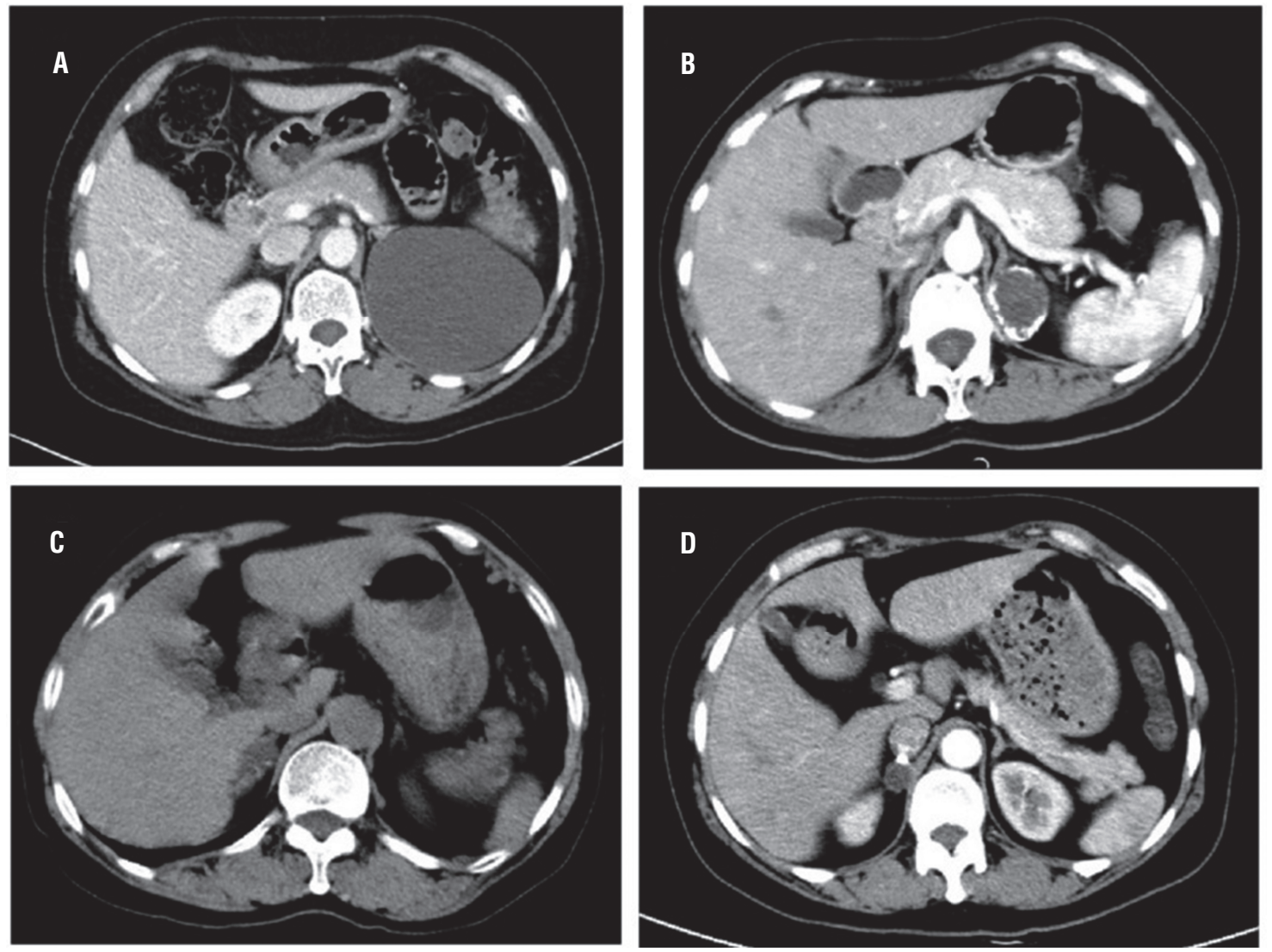

surgical techniques. Only one of the cases underwent FNA without sclerotherapy mediated by CT. Although FNA patients may avoid surgery when they present no clinical alteration in the punctured fluid, some experts argued that FNA has limited effect on determining cyst histopathology and the puncture fluid may reaccumulate $(2,3)$. Without histopathology diagnosis, it is hard to judge the cystic lesions malignancy. Surgical resection enabled us to obtain histopathologic diagnosis. Surgical resection were taken in our series in patients with the following characteristics: hormonally active adrenal cysts, cystic lesion greater than $4 \mathrm{~cm}$, smaller cysts enlarged at least by $1 \mathrm{~cm}$ per year, cysts with image changes like haemorrhage and calcification. Recently, multiple studies have described the minimally invasive approaches had shorter hospital stays, less blood loss, and enhanced cosmesis $(6,25-$ 27). In the present series, most open excisions were managed in the early time and great majority were submitted to laparoscopic excisions in recent years. Compared laparoscopic technology with open technology, the former has the advantage of a shorter operation time $(p<0.001)$, a shorter after-surgery hospital stay $(p=0.006)$ and enhanced cosmesis. According to our experience, we preferred open surgery for those re-operation 
Figure 2 - A) The endothelial cyst was lined by a single layer of flattened endothelial cells, $x 200$. B) The pseudocyst presented the absence of a single layer of flattened endothelial cells, x200. C) The adrenal lymphangiomatous cyst showed ectasia of lymphatic vessels in adrenal glands, $\mathbf{2 0 0}$. D) the pulmonary sequestration cyst was lined by benign pseudo-stratified ciliated columnar epithelium, x200.
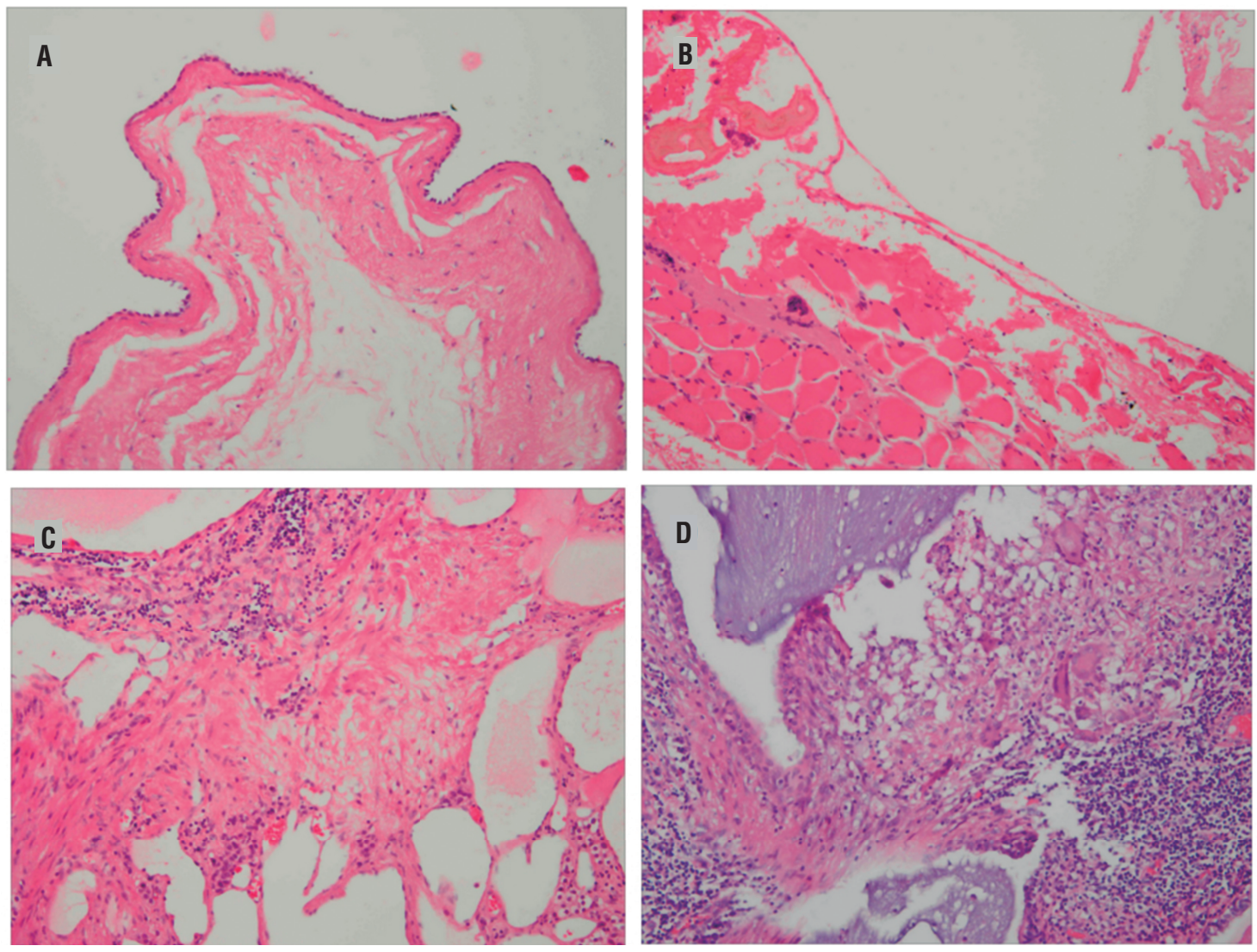

patients and cystic lesions with severe adhesion detected by imaging examination. We preferred retroperitoneal laparoscopy of the remaining patients with few complications being relatively safe. As operations performed from a single abdominal incision was popular, there have been no detailed randomized studies of ACL (4). Some authors chose laparoscopic adrenalectomy in all patients with adrenal cysts and other multiple centers prefer adrenal-sparing resections, even for functional cystic tumors (11). In our series, adrenal-sparing resections were managed in 37 $(80 \%)$ patients and adrenalectomies in $9(20 \%)$ patients. We preferred to preserve the adrenal gland except for the functional cysts or when the border between cysts and adrenal gland were unclear, accompanied with severe adhesion. One patient underwent laparoscopic adrenal-sparing resection presented a $2 \mathrm{~cm}$ cyst 3 months later. An open adrenalectomy was then performed and with long term follow-up no recurrence was found. The pathology showed the presence of a cystic adenoma.

\section{CONCLUSIONS}

Adrenal cysts are rare. Abdominal or flank pain and gastrointestinal symptoms are the main clinical manifestations. However, with the development of imaging techniques, adrenal cysts are always diagnosed incidentally. CT has advantages in detecting the cysts with haemorrhage, intracystic debris, calcification and mixed adrenal mass. Minimally invasive surgery offers 
equivalent efficacy to traditional open procedures, while providing a shorter operation time, shorter convalescence and improved cosmesis. However, surgical intervention is not recommended for asymptomatic patients with incidental cysts, for the fact that the pathological data of our series revealed no malignant cases. Patients after surgical resection should be followed up closely especially in functional cysts and when histopathology showed cystic tumor.

\section{ACKNOWLEDGMENT}

This research was funded by the National Natural

Science Foundation of China (Grant no. 30772171).

\section{ABBREVIATIONS}

ACL $=$ Adrenal cystic lesions

US $=$ ultrasound

$\mathrm{CT}=$ computed tomography

MRI = magnetic resonance imaging

FNA = percutaneous fine-needle aspiration

$\mathrm{CI}=$ confidence interval

\section{REFERENCES}

1. Tagge DU, Baron PL: Giant adrenal cyst: management and review of the literature. Am Surg. 1997; 63: 744-6.

2. Neri LM, Nance FC: Management of adrenal cysts. Am Surg. 1999; 65: 151-63.

3. Bellantone R, Ferrante A, Raffaelli M, Boscherini M, Lombardi CP, Crucitti F: Adrenal cystic lesions: report of 12 surgically treated cases and review of the literature. J Endocrinol Invest. 1998; 21: 109-14.

4. Major P, Pedziwiatr M, Małłok M, Ostachowski M, Winiarski M, Rembiasz K, et al.: Cystic adrenal lesions - analysis of indications and results of treatment. Pol Przegl Chir. 2012; 84: 184-9.

5. Kuruba R, Gallagher SF: Current management of adrenal tumors. Curr Opin Oncol. 2008; 20: 34-46.

6. El-Hefnawy AS, El Garba M, Osman Y, Eraky I, El Mekresh M, Ibrahim el-H: Surgical management of adrenal cysts: singleinstitution experience. BJU Int. 2009; 104: 847-50.

7. Wedmid A, Palese M: Diagnosis and treatment of the adrenal cyst. Curr Urol Rep. 2010; 11: 44-50.
8. Erickson LA, Lloyd RV, Hartman R, Thompson G: Cystic adrenal neoplasms. Cancer. 2004; 101: 1537-44.

9. Chien HP, Chang YS, Hsu PS, Lin JD, Wu YC, Chang HL, et al.: Adrenal cystic lesions: a clinicopathological analysis of 25 cases with proposed histogenesis and review of the literature. Endocr Pathol. 2008; 19: 274-81.

10. Erbil $Y$, Salmaslioglu A, Barbaros U, Bozbora A, Mete O, Aral $F$, et al.: Clinical and radiological features of adrenal cysts. Urol Int. 2008; 80: 31-6.

11. Castillo OA, Litvak JP, Kerkebe M, Urena RD: Laparoscopic management of symptomatic and large adrenal cysts. J Urol. 2005; 173: 915-7.

12. Pradeep PV, Mishra AK, Aggarwal V, Bhargav PR, Gupta SK, Agarwal A: Adrenal cysts: an institutional experience. World J Surg. 2006; 30: 1817-20.

13. Lal TG, Kaulback KR, Bombonati A, Palazzo JP, Jeffrey RB, Weigel RJ: Surgical management of adrenal cysts. Am Surg. 2003; 69: 812-4.

14. Stimac G, Katusic J, Sucic M, Ledinsky M, Kruslin B, Trnski $\mathrm{D}$ : A giant hemorrhagic adrenal pseudocyst: case report. Med Princ Pract. 2008; 17: 419-21.

15. da Silva EC, Viamontez F, Silva VS, Andrade A, Júlio Neto G, Gomes Cde P, et al.: Hemorrhagic adrenal cyst. Einstein (Sao Paulo). 2012; 10: 96-9.

16. Suh J, Heimann A, Cohen H: True adrenal mesothelial cyst in a patient with flank pain and hematuria: a case report Endocr Pathol. 2008; 19: 203-5.

17. Favorito LA, Lott FM, Cavalcante AG: Traumatic rupture of adrenal pseudocyst leading to massive hemorrhage in retroperitoneum. Int Braz J Urol. 2004; 30: 35-6.

18. Sanal HT, Kocaoglu M, Yildirim D, Bulakbasi N, Guvenc I, Tayfun C, et al.: Imaging features of benign adrenal cysts. Eur J Radiol. 2006; 60: 465-9.

19. Lockhart ME, Smith JK, Kenney PJ: Imaging of adrenal masses. Eur J Radiol. 2002; 41: 95-112.

20. Lunca S, Romedea NS, Roata C, Bouras G: Cystic lymphangioma of the adrenal gland. Chirurgia (Bucur). 2004; 99: 255-8.

21. Takeuchi $Y$, Ozaki $Y$, Suyama T, Sawada $Y$, Kuroda K, Nakajima $K$, et al.: A case of giant hemorrhagic adrenal pseudocystectomy with a flank incision. Hinyokika Kiyo. 2011; 57: 315-8.

22. Limaiem F, Korbi S, Jedidi S, Aloui S, Hassan F, Arfa N, et al.: Adrenal epithelial cyst: a case report. Pathologica. 2012; 104: 82-4.

23. Powell G, Burrows C, Houghton A, Otter M: An incidental peri-adrenal cystic lesion. BMJ Case Rep. 2012; 2012.

24. Liu L, Han P, Zhu Y, Gong J, Xu Y, Wei X, et al.: Intraabdominal pulmonary sequestration: a case report and literature review. Urol Int. 2012; 88: 121-4.

25. Nerli RB, Guntaka A, Devaraju S, Patil S, Hiremath MB: Adrenal cysts: Our laparoscopic experience. J Minim Access Surg. 2012; 8: 145-8. 
26. Disick Gl, Munver R: Adrenal-preserving minimally invasive surgery: update on the current status of laparoscopic partial adrenalectomy. Curr Urol Rep. 2008; 9: 67-72.
27. Lee J, El-Tamer M, Schifftner T, Turrentine FE, Henderson WG, Khuri S, Hanks JB, et al.: Open and laparoscopic adrenalectomy: analysis of the National Surgical Quality Improvement Program. J Am Coll Surg. 2008; 206: 953-9; discussion 959-61.

Correspondence address: Qiang Wei, MD Department of Urology, West China Hospital,

Sichuan University, 37, Guoxue Alley, Chengdu, Sichuan, 610041, P.R. China Fax: +86 288 542-2451 E-mail: weiqiang933@126.com 


\section{EDITORIAL COMMENT}

Adrenal cysts are uncommon lesions. Most of them are unilateral and can shown a remarkable variation in size. The reported incidence in post-mortem and clinical series has been $0.06-0.18 \%$ and up to $5.4 \%$, respectively $(1,2)$. We congratulate the authors for reporting in this manuscript one of the world's largest experiences on the treatment of adrenal cysts.

However, we would like to comment on the critical points of greatest controversy concerning adrenal cysts evaluation: 1) The preoperative differentiation between benign and malign lesions 2) The precise criteria for surgical indication 3) Is adrenal-sparing surgery feasible and appropriate? 4) The modern developments on minimally invasive techniques for adrenal surgery.

Primary adrenal gland cysts have traditionally been classified as pseudocysts, endothelial cysts (lymphangiomatous/angiomatous), and epithelial cysts and parasitic cyst. Although most of these lesions present a benign character, a wide histologic spectrum has been described (3). In this regard, adrenal cystic lesions have also been found harboring benign or malign adrenal cortical-medullar neoplasms foci on the cyst wall. Histopathological association with foci of adrenal cortical adenoma, cortical carcinoma or pheochromocytoma has already been previously reported. Parallel, adrenal solid neoplasms are also able to course presenting foci of cystic degenerative changes inside mimicking a primary adrenal cystic lesion. In addition, functionality can also be associated demanding endocrine investigation whereas metastatic tumors may eventually present as adrenal cysts complicating even more the clinical evaluation scenario (4). Therefore, attention must be given when treating these adrenal benign-appearing cysts, differentiating them from neoplastic or functional cysts.

In the modern era, adrenal cysts have been identified more incidentally and have shown a downward trend in diagnosis at symptomatic stages, especially due to an increased frequency of imaging studies performed and to developments achieved on imaging technology. In this context, CT scan is the preferred primary modality for imaging evaluation of the adrenal glands playing an important role in malignancy risk evaluation for adrenal solid or cystic lesions. Adrenal CT low risk features for solid lesions are described as less than $10 \mathrm{HU}$ on unenhanced CT, less than 30HU on enhanced CT, 10-minute delayed CT washout greater than 50\%, whereas water density up to 20HU, thin smooth walls and lack of enhancement are characteristics attributed to benign cystic lesions. Additionally, cysts wall thickness greater than $5 \mathrm{~mm}$, wall enhancement, thick rim or stippled central calcification may further suggests malignancy (5). Although cysts size is not considered criteria for malignancy, large cysts are related to an increased risk of massive hemorrhage, infection and mass effect symptoms. Cysts presenting hemorrhage or infection can lead to attenuation misinterpretation on CT scan what turns harder to rule out malignancy and therefore MRI scan can be helpful on differential diagnosis at this setting (6).

In this context, it is clear that adrenal cysts represent a heterogeneous group of pathological entities and that the precise preoperative diagnosis is a tough work. Consequently, adrenal cyst best management and treatment is often controversy, turning the management decision whereas surgical or conservative a paradigm. In general, surgical approach is recommended for all symptomatic or functional cysts, cysts greater than $5 \mathrm{~cm}$ due to risk of bleeding and for cysts with hemorrhagic or heterogeneous nature suggesting malignancy. On the other hand, although asymptomatic low suspicious small cysts can be followed conservatively, no surveillance protocol has been clearly defined (7). Management controversy relies on borderline lesions in which due to invasiveness and morbidity of open surgical adrenalectomy many cysts used to be treated conservatively (4). However, current developments on surgical field especially regarding to the minimally invasive techniques have changed this scenario, providing a remarkable reduction on surgical morbidity (8).

Currently, laparoscopic and more recently the robotic surgery have been the preferred minimally invasive approach for the treatment of adrenal lesions (9). Laparoscopic or robotic adre- 
nal-sparing resection is feasible allowing gland preservation when treating benign lesions (10). Advancements on laparoscopic surgical accesses have also been proposed for adrenalectomy. The laparoendoscopic single site (LESS) posterior retroperitoneoscopic access has risen as an emerging technique providing similar results to conventional laparoscopy with better aesthetics (11).

Percutaneous fine needle aspiration and cyst decortication were reported as treatment options, although no studies have investigated the potential risks of malignant spread due to cystic fluid leakage. Other important aspect is that cyst aspiration presents increased rates of recurrence and that complete surgical excision is the only option that allows cyst extensive sampling through histological examination (12).

In summary, minimally invasive techniques have determined a trend shift toward earlier surgical treatment of borderline cysts due to reduced surgical related morbidity.

\section{REFERENCES}

1. Castillo OA, Litvak JP, Kerkebe M, Urena RD: Laparoscopic management of symptomatic and large adrenal cysts. J Urol. 2005; 173: 915-7.

2. Bellantone R, Ferrante A, Raffaelli M, Boscherini M, Lombardi CP, Crucitti F: Adrenal cystic lesions: report of 12 surgically treated cases and review of the literature. J Endocrinol Invest. 1998; 21: 109-14.

3. Pradeep PV, Mishra AK, Aggarwal V, Bhargav PR, Gupta SK, Agarwal A. Adrenal cysts: an institutional experience. World J Surg. 2006; 30: 1817-20.

4. Wedmid A, Palese M. Diagnosis and treatment of the adrenal cyst. Curr Urol Rep. 2010; 11: 44-50.
5. Sanal HT, Kocaoglu M, Yildirim D, Bulakbasi N, Guvenc I, Tayfun C, Ucoz T: Imaging features of benign adrenal cysts. Eur J Radiol. 2006; 60: 465-9.

6. Guo YK, Yang ZG, Li Y, Deng YP, Ma ES, Min PQ, et al.: Uncommon adrenal masses: CT and MRI features with histopathologic correlation. Eur J Radiol. 2007; 62: 359-70.

7. Lal TG, Kaulback KR, Bombonati A, Palazzo JP, Jeffrey RB, Weigel RJ: Surgical management of adrenal cysts. Am Surg. 2003; 69: 812-4.

8. Tobias-Machado M, Lasmar MT, Zambon JP, Tristão R, Forseto PH Jr, Juliano RV, et al.: Laparoscopic adrenalectomy: a prospective study comparing transperitoneal and retroperitoneal approaches. Rev Assoc Med Bras. 2006; 52: 208-13.

9. Tobias-Machado M, Rincón Ríos F, Tulio Lasmar M, Tristão R, Hermínio Forseto P Jr, Vaz Juliano R, et al.: Laparoscopic retroperitoneal adrenalectomy as

10. a minimally invasive option for the treatment of adrenal tumors. Arch Esp Urol. 2006; 59: 49-54.

11. Walz MK, Peitgen K, Diesing D, Petersenn S, Janssen OE, Philipp T, et al.: Partial versus total adrenalectomy by the posterior retroperitoneoscopic approach: early and longterm results of 325 consecutive procedures in primary adrenal neoplasias. World J Surg. 2004; 28: 1323-9.

12. Tobias-Machado M, Matos $P$, Juliano $C$, Junior R, Carlos A, Pompeo A. Adrenalectomia retroperitoneal posterior single port em paciente com feocromocitoma. Congresso Brasileiro de Urologia - 2013, Resumos Pubicados em Anais de Eventos 2013.

13. Neri LM, Nance FC. Management of adrenal cysts. Am Surg. 1999; 65: 151-63.

\author{
Igor Nunes-Silva, $M D \&$ \\ Marcos Tobias-Machado, MD \\ Section of Urology \\ ABC Medical School \\ São Paulo, SP, Brazil \\ E-mail: tobias-machado@uol.com.br
}

